\title{
On a New Class of Univariate Continuous Distributions that are Closed Under Inversion
}

\author{
Saleha Naghmi Habibullah \\ Kinnaird College for Women \\ Lahore, Pakistan \\ Email: salehahabibullah@hotmail.com \\ Munir Ahmad \\ National College of Business Administration \\ \& Economics, Lahore, Pakistan \\ Email:drmunir@brain.net.pk
}

\begin{abstract}
Inverted probability distributions find applications in various real - life situations including econometrics, survey sampling, biological sciences and life - testing. Closure under inversion implies that the reciprocal of a continuous random variable $X$ has the same probability function as the original random variable, allowing for a possible change in parameter values. To date, only a very few probability distributions have been found to possess the closure property.
\end{abstract}

In this paper, an attempt has been made to generate a class of distributions that are closed under inversion, and to develop some statistical properties of this class of distributions.

Keywords: Inverted probability distribution, Closure under inversion

\section{Introduction}

Inverted probability distributions find applications in various real - life situations including econometrics, survey sampling, biological sciences, engineering sciences, and, most prominently, in life - testing. As such, various authors have derived a variety of inverted distributions, and have developed their statistical properties.

Closure under inversion implies that the reciprocal of a continuous random variable $X$ has the same probability function as the original random variable, allowing for a possible change in parameter values. In case the parameter values are identical to those of the original distribution, the random variable $X$ (and its reciprocal) will be said to be Strictly Closed Under Inversion.

To date, only a very few probability distributions have been found to possess the closure property. For example, the Cauchy $(0,1)$ distribution is closed under inversion in the strict sense, whereas the $\mathrm{F}\left(v_{1}, v_{2}\right)$ distribution is closed in the generalized sense.

In this paper, an attempt has been made to generate a class of distributions that are closed under inversion, and to establish some of the fundamental properties of this particular class of distributions. 


\section{Closure Under Inversion}

\section{Definition}

A probability density function $f(x)$ will be said to be closed under inversion if the form of the probability density function of $1 / X$ is the same as that of $f(x)$. In case the parameters of the inverted distribution are identical to those of the original distribution, the random variable $X$ (and its reciprocal) will be said to be strictly closed under inversion.

\section{A Class of Distributions that are Strictly Closed Under Inversion}

With reference to the development of a class of distributions that are Strictly Closed Under Inversion, we present the following theorem:

\section{Theorem 3.1}

Every function of the form

$$
f(x)=k[M[w(x) \cdot w(1 / x)]] / x
$$

defined on $(a, 1 / a), 0<a<1$

where

(i) $\mathrm{k}$ is a non - zero real number,

(ii) $w$ represents an algebraic function of $x$ \&

(iii) $M$ represents an algebraic function of $w(x) \cdot w(1 / x)$

such that

a) $f(x) \geq 0$ over its domain, and

$$
1 / a
$$

(b) $\$ f(x) d x=1)$

a

represents a continuous probability distribution that is Strictly Closed Under Inversion.

Proof

Let $Y=1 / X$;

Then

modulus of $d x / d y=1 / y^{2}$

When $x \rightarrow a, y->1 / a$

When $x \rightarrow 1 / a, y->a$ 
Hence

$h(y)=k[M[w(1 / y) \cdot w(y)]] y \cdot 1 / y^{2}=k[M[w(y) \cdot w(1 / y)]] / y, \quad a<y<1 / a$

------ exactly the same form as that of $f(x)$

Hence the function given by (3.1) is Strictly Closed Under Inversion.

As far as the point regarding $f(x)$ being a proper pdf is concerned, any function of the form given by (3.1) will be a proper pdf as long as $f(x)>0$ over its domain, and the integral of the function over its domain is convergent.

\section{Alternative Proof}

If the function $f(x)$ is Strictly Closed Under Inversion, then it satisfies the functional equation

$$
\begin{array}{r}
f(x)=f(1 / x) / x^{2} \\
\text { or } \quad x f(x)=f(1 / x) / x
\end{array}
$$

Now eq (3.1) can be written as

$$
x f(x)=k[M[w(x) \cdot w(1 / x)]]
$$

Replacing $x$ by $1 / x$, we obtain

$$
\begin{aligned}
& f(1 / x) / x=k[M[w(1 / x) \cdot w(x)]] \\
& =k[M[w(x) \cdot w(1 / x)]]
\end{aligned}
$$

Hence we have $x f(x)=f(1 / x) / x$

$$
\text { or } f(x)=f(1 / x) / x^{2}
$$

Hence the function $f(x)$ given by (3.1) is Strictly Closed Under Inversion.

\section{Remarks}

1. In the above, if we let $\mathrm{a}->0^{+}$then the domain $(\mathrm{a}, 1 / \mathrm{a})$ tends to $(0$, infinity).

2. If we let $a->1$, then $1 / a->1$ and $f(x)$ is degenerate. (In other words, if $a->1$, $f(x)$ is a one - point distribution located on $x=1$.)

\section{Examples}

The class of SCUI distributions given by (3.1) encompasses a variety of probability density functions, some of which are presented in Table 1: 
Table 1: Some distributions belonging to the class of

Probability Distributions Given by Equation (3.1)

\begin{tabular}{|c|c|c|}
\hline $\mathbf{S} \#$ & Distribution & Remarks \\
\hline 1. & $\mathrm{f}(\mathrm{x})=1 / 2 \mathrm{ax} \quad \mathrm{e}^{-\mathrm{a}}<\mathrm{x}<\mathrm{e}^{\mathrm{a}}, \mathrm{a}>0$ & $\begin{array}{l}\text { Here } \\
\qquad \begin{array}{l}k=1 / 2 a \\
w(x) \text { could be any function of } x\end{array} \\
\& \quad M=[w(x) \cdot w(1 / x)]^{0}=1\end{array}$ \\
\hline 2. & $\begin{array}{l}g(y)=\left(\frac{1}{y}\right)\left[\frac{1}{\sigma \sqrt{2 \pi}} e^{-\frac{1}{2}\left[\frac{\ln y}{\sigma}\right]^{2}}\right], \\
0<\mathrm{y}<\infty, \sigma>0\end{array}$ & $\begin{array}{l}\text { Here } \\
\quad k=1 / \text { sigma [sq rt of } 2 p i] \\
\quad w(y)=\ln y \\
\text { so that } w(1 / y)=-\ln y \\
\text { and } \\
w(y) w(1 / y)=-(\ln y)^{2} \\
\& \\
M=\exp \left\{\left[w(y) \cdot w(1 / y) / 2 \text { sigma }^{2}\right]\right.\end{array}$ \\
\hline 3. & $\begin{array}{l}\text { The well - known } F \text { distribution } \\
\text { with } \mathrm{v} 1=\mathrm{v} 2=\mathrm{v} \\
\text { i.e. } \\
\mathrm{g}(\mathrm{x})=\frac{\Gamma(\mathrm{v})}{\left[\Gamma\left(\frac{v}{2}\right)\right]^{2}} \mathrm{x}^{\mathrm{v} / 2^{-1}}(1+\mathrm{x})^{-v}, \quad 0<\mathrm{x}<\infty\end{array}$ & $\begin{array}{l}\text { Here } \\
\qquad \begin{array}{l}\mathrm{K}=\frac{\Gamma(v)}{[\Gamma(v / 2)]^{2}} \\
\& \quad \mathrm{w}(\mathrm{x})=\mathrm{x}^{v / 4}(1+\mathrm{x})^{-v / 2} \\
\quad \mathrm{M}=[\mathrm{w}(\mathrm{x}) \cdot \mathrm{w}(1 / \mathrm{x})]\end{array}\end{array}$ \\
\hline
\end{tabular}

4. Some Properties Pertaining to the Quantiles of Scui distributions given by ( 3.1 )

\section{Theorem}

For every pdf belonging to the class of SCUI distributions given by (3.1) above, the $(1-q)^{\text {th }}$ quantile is the reciprocal of the qth quantile i.e.

$$
x_{1-q}=1 / X_{q} \quad \text { where } 0<q<1
$$

\section{Proof}

Let $f(x)$ be a continuous SCUI distribution defined on [a, 1/a ], $0<a<1$.

Then $f(x)$ satisfies the functional equation

$$
f(x)=f(1 / x) / x^{2} \text { for all } x \text { belonging to }[a, 1 / a] .
$$


Hence

$$
\begin{array}{cl}
x_{q} & X_{q} f(x) d x= \\
a & =
\end{array}
$$

Now, in the RHS of eq. (2), let $Y=1 / X$ so that $X=1 / Y$.

When $x=a, y=1 / a$

When $x=x_{q}, y=1 / x_{q}$

$d x=-d y / y^{2}$

Hence, eq (2) becomes

$$
\begin{array}{cl}
X_{q} & 1 / X_{q} \\
q=\$ f(x) d x & =-\$\left[y^{2} f(y) / y^{2}\right] d y \quad a \\
1 / a \quad 1 / a & 1 / a \\
& =\$ f(y) d y=\$ f(x) d x \quad \text { (dummy variable) } \\
1 / X_{q} \quad 1 / X_{q}
\end{array}
$$

But

$$
q=\begin{array}{cc}
x_{q} & 1 / a \\
a & 1 / X_{q}
\end{array}
$$

means that

Hence proved.

$$
1 / x_{q}=x_{1-q}
$$

\section{Corollary No. 1}

For every pdf belonging to the class of SCUI distributions given by eq. (3.1) above, the median is equal to unity i.e.

$$
\mathrm{X}=\mathrm{X}_{0.5}=1
$$

\section{Proof}

We know that, for every pdf belonging to the class of SCUI distributions given by eq. (3.1) above,

$$
\begin{aligned}
x_{1}-q & =1 / X_{q} \\
\text { where } & <q<1
\end{aligned}
$$

Putting $q=0.5$, we obtain

$$
\begin{aligned}
X_{0.5}= & 1 / X_{0.5} \\
= & {\left[X_{0.5}\right]^{2}=1 } \\
= & X_{0.5}=1 \\
& (\text { since } X \text { a positive random variable). }
\end{aligned}
$$




\section{Remark}

The converse of this result is not generally true. There do exist continuous distributions that are defined on ( 0 , infinity) and the median of which is unity but which are not Strictly Closed Under Inversion.

\section{Corollary No. 2}

For every pdf belonging to the class of SCUI distributions given by eq. (3.1) above, the area under the curve between $X_{q}$ and $1 / X_{q}$ is equal to $1-2 q$ i.e.

$$
P\left[X_{q}<X<1 / X_{q}\right]=1-2 q
$$

\section{Proof:}

The proof is simple.

\section{Remark}

This result is somewhat comparable with the well - known result that, for a normal distribution with mean Mu and standard deviation Sigma:

$$
\begin{aligned}
& \mathrm{P}[\mathrm{Mu}-\text { Sigma }<\mathrm{X}<\mathrm{Mu}+\text { Sigma }]=0.6826 \\
& \mathrm{P}[\mathrm{Mu}-2 \text { Sigma }<\mathrm{X}<\mathrm{Mu}+2 \text { Sigma }]=0.9544 \\
& \mathrm{P}[\mathrm{Mu}-3 \text { Sigma }<\mathrm{X}<\mathrm{Mu}+3 \text { Sigma }]=0.9973
\end{aligned}
$$

\section{Some General Remarks}

\section{Remark No. 1}

All of the above is true under Regularity Conditions such as absolute continuity, absolute differentiability, etc.

\section{Remark No. 2}

The class of distributions of non - negative random variables given in this paper is not exhaustive. There exists at least one probability density function that extends from-infinity to infinity and is closed under inversion i.e. the Cauchy $(0,1)$ distribution.

\section{References}

1. Ahmad, M. and Sheikh, A.K. (1981). Reliability Computation for Bernstein Distribution Strength and Stress. Submitted to the $10^{\text {th }}$ Pak. Statistical Conference held in Islamabad, Pakistan. 
2. Ahmad, M. and Sheikh, A.K. (1983). Some Estimation Problems of a Class of Inverted Distributions. Contributed Paper, $44^{\text {th }}$ Session of the International Statistical Institute, Madrid, Espana.

3. Ahmad, M. and Sheikh, A.K. (1984). Bernstein Reliability Model: Derivation and Estimation of Parameters. Reliability Engineering, Vol. 8, pp 131-148.

4. Bartholomew, A.J., (1957). A Problem in Life Testing. JASA, Vol. 52, pp 350-351.

5. Cobb, L. (1980). The Multimodal Exponential Families of Statistical Catastrophe Theory. Statistical Distributions in Scientific Work, Vol. 4, pp. 69 - 90.

6. Deming, W.E. (1950). Some Theory of Sampling. John Wiley and Sons, New York, pp 449-454.

7. Druzhinin, G.V. (1963). Reliability of Automatic Devices, Energiya.

8. Epstein, B. and Sobel, M., (1953). Life Testing. JASA, Vol. 48, pp 486502.

9. Epstein, B. and Sobel, M. (1954). Some Theorems Relevant to Life Testing. The Annals of Mathematical Statistics, Vol. 25, pp 373-381.

10. Habibullah, S. N. (1987). On the Modes of the Inverted Bivariate Normal Distribution. Pakistan Journal of Statistics, Vol., No., pp.

11. Kazi, M.H., Ahmad, M. and Sheikh, A.K. (1982). On a Two Parameter Bernstein Probability Distribution. Submitted.

12. Kordonsky, K.B. and Fridman, Y.M. (1976). Certain Questions Concerning the Probabilistic Description of Fatigue Longevity (survey),

(in Russian, Zavodskaya Laboratoriya, Vol. 7, pp 829-847),

(in English: Industrial Laboratory, INDLAP 42(7), 1001-1186, Consultants Bureau, New York).

13. Pronikov, A.S. (1973) Calculation of Reliability Indices in Sequential (wear based) Failures. Nedezhnosti Kontrol Kachestra, No. 2 (in Russian).

14. Pronikov, A.S. (1973). Dependability and Durability of Engineering Products. John Wiley and Sons, New York.

15. Sheikh, A.K. and Ahmad, M. (1982). A Reliability Model for a Non-linear Damage Process. $19^{\text {th }}$ Annual Meeting of the Society of Engineering Sciences, Rolla, Missouri.

16. Sheikh, A.K., and Ahmad, M., (1983). Material Damage Processes and Reliability Models. Contributed Paper, $44^{\text {th }}$ Session of the International Statistical Institute, Madrid, Espana.

17. Sheikh A.K. and Ahmad, M. (1984). On the Bernstein Distribution with Engineering Applications. Technical Report, UPM Grant No. ME/Modelling/46.

18. Seshadri, V. (1965). On Random Variables which have the Same Distribution as their Reciprocals. Canadian Mathematical Bulletin, Vol. 8, No. 6, pp 819-824. 
19. Vysokovskii, E.S. (1966). Reliability of Tools Used on Semi Automatic Lathes. Russian Engineering Journal, Vol. XLVI, No. 6, pp 46-50.

20. Vysokovskii, E.S. (1970). Reliability of Cutting Tools in Automated Production. Russian Engineering Journal, Vol. No. 3, pp 63-67.

21. Weibull, W. (1939). A Statistical Theory of Strength of Materials. Ing. Vetenskaps Akad. Handl., Stockholm, Vol. 73. 